\title{
PREVALENCE AND MOLECULAR CHARACTERIZATION OF GUMBORO VIRUS IN CHICKEN FARMS IN ISMAILIA
}

\author{
NEVEN RAMZY*; SHAHERA ABDEL-FATTAH ${ }^{* *}$ and ABDEL-DAYEM M.** \\ * Institute of Animal Health Research, Dokki, Giza, Ismailia branch. \\ ** Department of Virology, Faculty of Vet. Medicine, Suez Canal University.
}

Email: nevenramzy268@yahoo.com

Assiut University Email: www.aun.edu.eg

\section{ABSTRACT}

Received at: 5/3/2015

Accepted: 9/5/2015
Infectious bursal disease (IBD, Gumboro disease) is a worldwide immunosuppressive disease of chickens in their first weeks of life. IBD is one of the most economically important diseases that affects commercially produced chickens. Vaccination programmes with inactivated and live attenuated viruses have been used to prevent IBD. Some of the vaccines may not induce full protection against the very virulent IBDV strains and antigenic variants. In this study, 20 farms were represented for a prevalence study of the disease in Ismailia governorate, Egypt (IBDV-Ismailia-isolate-2014). Isolates from bursal samples of commercial broiler farms exhibiting some clinical and postmortem signs with poor performance were used for Reverse transcription (RT) - polymerase chain reaction (PCR) assay. RTPCR was used to amplify a 620-bp product within the hypervariable region of the IBDV VP2 gene. Three farms out of $20(15 \%)$ were positive. The positive samples were subjected for nucleotide sequence analysis (265 nucleotide in hyper variable region of VP2). Nucleotide and amino acid sequence analysis and blast indicated that Ismailia isolateshave close relationships between the previously isolated Egyptian IBDVs and they are clustered together (with 99-100\% identity) while other classic attenuated vaccine strains (Bursavac and Bursine) were grouped in another group (with $92.45 \%$ and $91.32 \%$. identity respectively). This result guides us to review the vaccination programs against IBDV in Egypt and better selection of vaccines that canimprove the immune response against the infection.

Key words: Gumboro virus, Chicken farms, Ismailia, Egypt.

\section{INTRODUCTION}

Infectious bursal disease (IBD) is a highly contagious immunosuppressive disease of young chickens caused by infectious bursal disease virus (IBDV) (Wang et al., 2011). It is a double-stranded RNA virus, genus Avibirna virus of the family Birnaviridae. IBDV was first recognized as a disease entity in "Gumboro", district of Delaware State in USA at 1957 by Cosgrove while it was first recorded in Egypt by ElSergany at 1974 (Cosgrove, 1962; El-Sergany et al., 1974).

The IBDV genome is divided into segments $\mathrm{A}$ and $\mathrm{B}$ : segments A $(3.4 \mathrm{~kb})$ and $\mathrm{B}(2.8 \mathrm{~kb})$. The large segment A encodes 4 viral proteins, the two capsid proteins VP2 (48 $\mathrm{kDa})$ and VP3 $(32-35 \mathrm{kDa})$, the viral protease VP4 $(24 \mathrm{kDa})$ and a nonstructural protein VP5 (17-21 kDa), while the smaller segment
B encodes VP1 (90 kDa), an RNA-dependent RNA polymerase. Expression/deletion studies have shown VP2 aa positions 206 to 350 to represent a major conformational and neutralizing antigenic domain called hyper variable region (HVR) (Xu et al., 2011) which includes the most variable region important for cell antigenic and pathogenic variation. Most exchanges of amino acid residues in VP2 occur in the four hydrophilic loops of the viral capsid (Coulibaly et al., 2010).

Four pathotypes, attenuated, virulent, antigenic variant and very virulent have been identified ( $\mathrm{Li}$ et al., 2009). Serotype 1 IBDV causes economically significant immunosuppressive disease in young chickens. Within serotype 1, two subtypes are identified, classic and variant (Wu et al., 2007a). Because of the huge economic losses caused by this virus to poultry industry, vaccination has therefore been applied intensively to control the infection 
(Kusk et al., 2005). Effective vaccination depends on rapid and accurate diagnosis of the subtype present in a flock because vaccines based on the classic subtype of IBDV can fail to protect against challenge with a variant subtype (Wu et al., 2007b).

Some studies suggested that there are some mutations which do not change key amino acid residues in the domains which are essential for its virulence. It suggests that a virulent IBDV strain could maintain its virulence for a long period in the same chicken farm and the strain is highly stable under normal environmental conditions (Li et al., 2009). Rapid and sensitive investigation for this virus in recent years is based on molecular diagnostic methods by reverse transcription-polymerase chain reaction (RT-PCR) for amplification of the IBDV VP2 gene. Conventional RT-PCR has been useful in detecting IBDV serotypes and, to a lesser extent, differentiating IBDV subtypes. Conventional RT-PCR, amplifying the VP2 hypervariable region, in combination with DNA sequencing of the PCR product, can differentiate classic, variant, and vvIBDV strains because variant and VvIBDV have characteristic nucleotide and amino acid substitutions. These methods potentially allow for more rapid, sensitive, and specific detection and differentiation of IBDV classic, very virulent, and variant subtypes. This approach is a valuable tool for molecular epidemiological studies on IBDV (Islam et al., 2012). The comparative analysis will indicate that if these viruses are genetically close to the vvIBDVs or classical strains (Singh et al., 2012).

The infectious bursal disease virus continuous to be a serious problem in Egypt, In this study, 20 broiler farms in Ismailia Governorate over the period 20122014 were investigated in an attempt to molecular characterize IBDV using RT-PCR, isolation, sequence analysis of HVR part of the VP2 gene, to provide information about the existence of VvIBDV in Ismailia region.

\section{MATERIALS and METHODS}

\section{Broiler flocks}

Two hundred bursal samples were collected from twenty 3-8 weeks broiler chicken farms, located in Ismailia governorate (10 samples from each farm), during the period of 2012- 2014. Clinical signs observed were diarrhea, swelling in hock joint, dullness and congestion in comb, with sudden onset of high mortalities. P/M findings included enlarged congested bursa, peticheal hemorrhages on the leg and breast muscles, congested and enlarged liver.

\section{Clinical samples:}

Bursal samples were obtained from different poultry farms in Ismailia. Each bursal sample was homogenized in sterile phosphate- buffered saline and a $10 \%$ tissue suspension was prepared following standard procedures. The suspension was clarified by centrifugation. An aliquot of the tissue suspension was used for virus isolation and extraction of RNA as described below.

\section{Reverse transcription and genetic amplification by polymerase chain reaction (RT-PCR):}

RNAs were extracted from the bursal homogenates [one part of each bursa sample disrupting in sterile saline (1:1)] using QiAmp Viral RNA Mini kit (Qiagen GmbH, Hilden, Germany) according to the manufacturer's instructions. A set of primers were used for the RT-PCR reaction and for the subsequent sequence analysis using forward [AUS GU: 5'-TCA CCG TCC TCA GCT TAC CCA CAT C-3'] and reverse PCR primers [AUS GL: 5'-GGA TTT GGG ATC AGC TCG AAG TTG C-3'] (Bayliss et al., 1990) for amplification of a $620 \mathrm{bp}$ fragment within IBDV on VP2 gene. The reaction mixture is $50 \mu \mathrm{l}$ contained $10 \mu 1$ of extracted RNA, $10 \mu 1$ of $5 x$ RTPCR buffer, $2 \mu 1$ primer F, $2 \mu 1$ primer R, , $2 \mu 1 \mathrm{dNTP}$ mix containing $400 \mu \mathrm{M}$ each dATP, dGTP, dCTP, dTTP, and $2 \mu \mathrm{l}$ of Qiagen OneStep Enzyme Mix.

A fragment of $620 \mathrm{bp}$ of the 5'-noncoding region was amplified by PCR thermo cycling using (T3 Biometra- Germany) as follows: 20 minutes at $50^{\circ} \mathrm{C}$ (RT reaction); $95^{\circ} \mathrm{C}$ for 15 minutes (initial PCR activation); 39 three-step cycles of $94^{\circ} \mathrm{C}$ for $30 \mathrm{sec}$ (denaturation), $59^{\circ} \mathrm{C}$ for $40 \mathrm{sec}$ (annealing) and $72^{\circ} \mathrm{C}$ for 1 minute (extension); then $72^{\circ} \mathrm{C}$ for 10 minutes (final extension). After amplification, $5 \mu \mathrm{l}$ of PCR products were analyzed by electrophoresis on a $1.5 \%$ agarose gel containing ethidium bromide with final concentration of $0.5 \mu \mathrm{g} / \mathrm{ml}$ at $95 \mathrm{~V}$ for 30 minutes in 1x TBE buffer, against GeneRuler ${ }^{\mathrm{TM}} 100$ bp Plus DNA Ladder (Fermentas). Images of the gels were photographed on BioDoc Analyze Digital Systems (Biometra, Germany).

\section{Sequence analysis of VP2 gene:}

Gel containing RNA band of the expected size was excised and purified with the QIAquick Gel Extraction Kit (Qiagen, USA) according to the manufacturer instruction. The purified PCR products were sequenced directly using the ABI PRISM® BigDyeTM Terminators v3.1 Cycle Sequencing Kit (Applied Biosystems, Foster City, CA, USA) and the ABI PRISM ${ }^{\circledR} 3130$ genetic analyzer (Applied Biosystems) with $80 \mathrm{~cm}$ capillaries, assembly of the consensus sequences and alignment trimming was performed with the BLAST tool. Used for comparison with other Egyptian viruses from the Genbank and in comparison with other international strains.

The sequences were edited with SeqScape_Software Version 2.5 (Applied Biosystems), assembly of the consensus sequences and alignment trimming was 
performed with the Lasergene DNASTAR group of programs (DNASTAR Inc., Madison, WI), Using Clustal V method. The phylogram was drawn using also MEGA 5 software. The alignment of the viruses in the study was done using MEGA 5 and BioEdit programs. Egyptian viruses and other international reference strains from the Genbank and were available from the National Center for Biotechnology Information (NCBI) infectious bursal disease virus's resource. Finally the identity percent and divergence between all viruses was carried out.

\section{RESULTS}

Affected chickens from 20 flocks showed congested bursa, severepetechial hemorrhages on breast and thigh muscles, kidney congestion and enlargement with mottled appearance and bones were bended and easily brocken, with history of high mortalities (11$15 \%)$. The rest of examined flocks showed dehydration with slight muscular congestion and slightly enlarged bursa.

\section{Detection of IBDV by RT-PCR}

Out of 20 broiler chicken farms bursal samples tested with RT-PCR, 3 farms (15\%) were positive. All RTPCR positive samples showed specific bands at 620 bp on agarose gel (Figure 1).

\section{Post mortem findings}

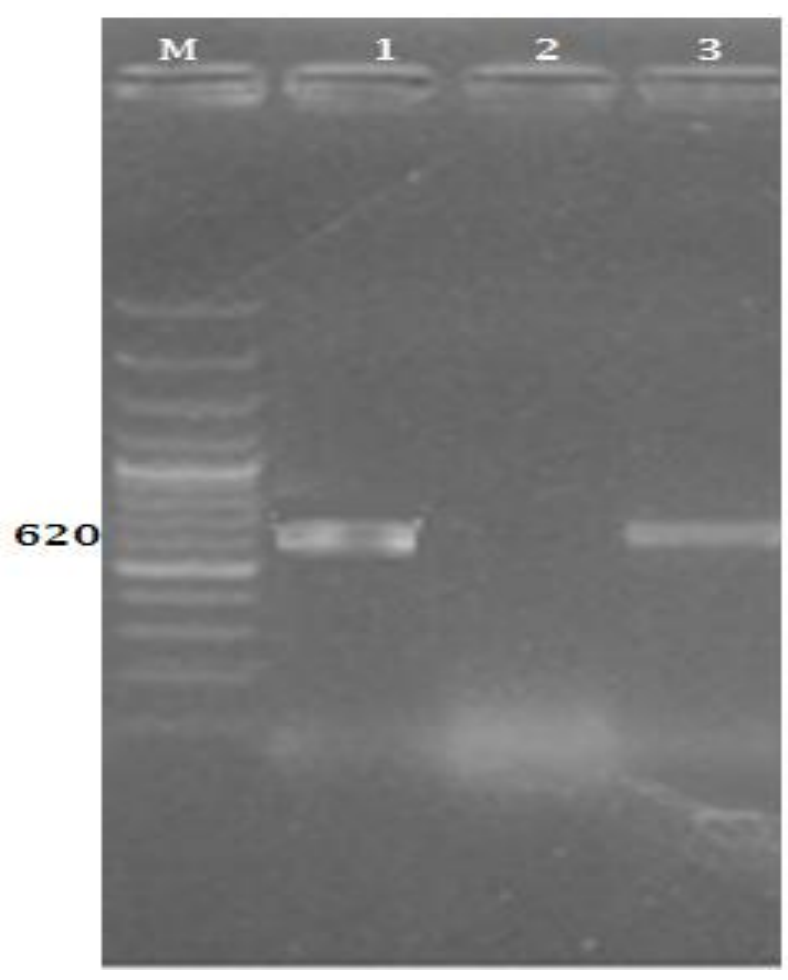

Figure (1):- Gel electrophoresis of Rt-PCR showing 620 bp band with positive control (Lane 1) and positive sample (lane 3) and no band was observed in negative control (lane 2). M=100 bp ladder.

\section{Results of sequence analysis}

Regarding to nucleotide sequence analysis of IBDVIsmailia-isolate-2014, a total of 265 nucleotide sites in hyper variable region (VP2) of a segment of the viral gene was characterized for the whole dataset and compared with the IBDV sequences in Gen Bank database using a BLAST search via the National Center of Biotechnology Information (USA). BLAST report results revealed that, IBDV_Ismailia-2014 isolate shared significant similarity at the nucleotide level ranged from $99 \%$ to $100 \%$ with other Egyptian vvIBDV (Giza 2008 and Giza 2000) as shown in figure (2) and table (1).
There is only one base difference (C32G) between IBDV_Ismailia-2014 isolate and Giza 2008, IBDV_S10-2013, IBDV_S10-2013 and IBDV_S102013 isolates with $99.62 \%$ identity. Also when compared with other circulating Egyptian strains as S1-2012, S2-2012, S3-2012, S4-2012 and S11-2013 our isolates showed very high similarity ranged from $99 \%$ to $100 \%$ but when compared with vaccinal strains as Bursavac and Bursine it showed $92.45 \%$ and $91.32 \%$ identity respectively. 


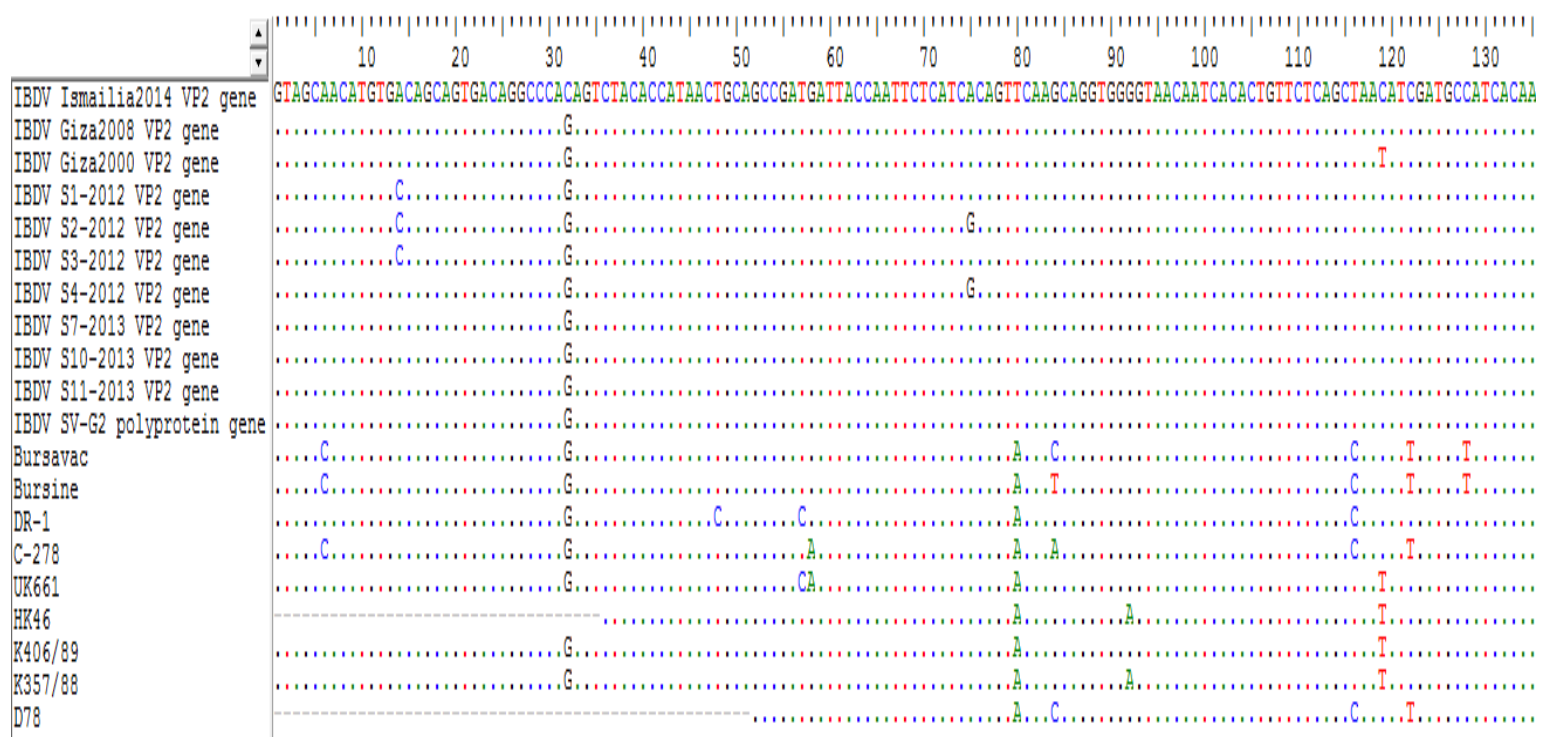

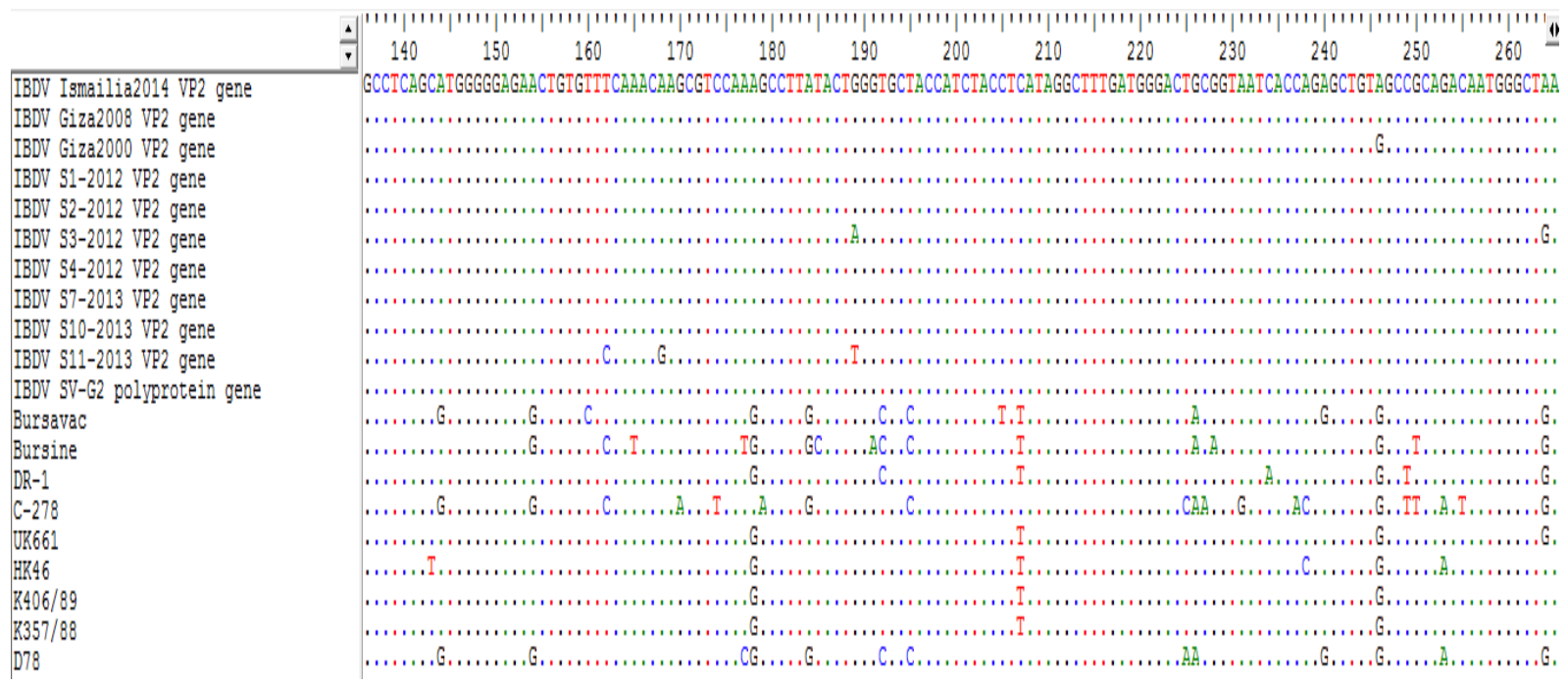

Figure (2): Nucleotide sequences of different Egyptian IBDV strains compared with other strains in GenBank. The horizontal line represents the sequence limits of VP2 gene. Dots indicate identical sequences

\section{Amino acid alignment of VP2 sequence of isolated IBDV}

As shown in figure (3) and table (1) from 88 amino acids there is only one amino acid difference (T11R) between IBDV_Ismailia-2014 isolate and other Egyptian circulating strains as Giza 2008, S4-2012, S7-2013, S10-2013, S11-2013 and SV-G2 isolates with identity percent $98.86 \%$ and only two amino acid differences (T11R and D5A) with other Egyptian strains as Giza 2000, S1-2012, S2-2012 and S3-2012 with identity percent $97.73 \%$ but when compared with classic attenuated vaccine strains (Bursavac and Bursine) showed 12 and 14 amino acid differences with percent of identity $86.36 \%$ and $84.10 \%$ respectively. 
$\begin{array}{lll}20 & 30 & 40\end{array}$

\begin{tabular}{|c|c|c|c|c|c|c|c|c|c|c|c|c|c|c|c|c|c|c|c|c|c|c|c|c|c|c|c|c|c|c|c|c|c|c|c|c|c|c|}
\hline lame & V A & & & & & & & & & & & & & & & & & & & & & & & & & & & & & & & & & & & & & I $P$ \\
\hline 1. IBDV Ismaila2014 VP2 gene & . & & & & & & & & & & & & & & & & & & & & & & & & & & & 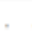 & . & & . & & & & . & . & . . & \\
\hline IBDV Gza2008 & & & & & & & & & R & & & & & & & & & & & & & & & & & & & & & & & & & & & & & \\
\hline IBDV Gza2000 V & & & & & & & & & R & & & & & & & & & & & & & & & & & & & & & & & & & & . & 1 & & \\
\hline 4. IBDVS & & & A & A & & & & & $\mathrm{R}$ & & & & & & & & & & & & & & & & & & & & & & & & & & & & & \\
\hline IBDI & . & . & & A & & & . & & $\mathrm{R}$ & & . & & & . . & & & & & & . & & . & & & & & & - & & & & & . & & . & . & $\therefore$ & \\
\hline $\mathrm{BD}$ & . & & & A & & & & & $\mathrm{R}$ & & . & & & - . & & . & & . & & . & & . & & . & & . & & . & & . & . & & . & & . & & . . & . \\
\hline & . & & . & . & & & & & $\mathrm{R}$ & & & & & & & & & & & & & & & & & & & & & & & & & & & & & \\
\hline $\mid \mathrm{BD}$ & . & & & & & & & & $R$ & & & & & & & & & & & & & & & & & & & & & & & & & & & & & \\
\hline 9. IBD & & & & & & & & & R & & & & & & & & & & & & & & & & & & & & & & & & & & & & & \\
\hline 10. IBDV & & . & & & & & & & $\mathrm{R}$ & & . & & & & & . & & & & . & & . & & & & & & & & & & & . & & $\cdot$ & & & \\
\hline 11. IBDV SV-G2 & . & & & & & & & & R & & . & & & & & . & & . & & . & & . & & & & . & & - & & & & & . & & . & & . . & . \\
\hline 12. Bursavac & . & . & & . & & & & & R & & & & & & & & & & & & & & & $Y$ & N & & & & & & & & & & $P$ & .1 & L. & $\mathrm{L}$ \\
\hline 13. Bu & . & & & & & & & & $\mathrm{R}$ & & & & & & & & & & & & & & & $\gamma$ & N & & & & & & & & & & $P$ & .1 & L. & $\mathrm{L}$ \\
\hline 14. D & . & & & & & & & & $\mathrm{R}$ & & & & & & & & & & & & & & & $Y$ & & & & & & & & & & & $P$ & & & \\
\hline $15 . \mathrm{C}$ & & . & & & . & & & & $R$ & & & & & & & & N & & & & & & & & & & & & & & & & 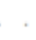 & & $P$ & & L & . \\
\hline 16.0 & . & & & & & & & & $\mathrm{R}$ & & . & & & & & . & N & . & & 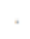 & & & & & & . & & & & & & & . & & . & 1 & . & . \\
\hline 17. $\mathrm{HK}$ & . . & . & $\cdot$ & $\cdot$ & . & . & . & $\cdot$ & & $?$ & . & & & & & & & & & & & & & $Y$ & & & & $E$ & & & & & & & & 1 & & \\
\hline 18. K406/89 & . & & & & & & & & $\mathrm{R}$ & & & & & & & & & & & & & & & & & & & & & & & & & & & 1 & & \\
\hline 19. K34 & . & & & & & & & & $R$ & & & & & & & & & & & & & & & $Y$ & & & & & & & & & & & & 1 & & \\
\hline 20.078 & . & . & $\cdot$ & $\cdot$ & & & & . & $\cdot$ & . & $\cdot$ & - & & & & & & & & & & & & & & & & & & & & & & & $P$ & & L. & \\
\hline
\end{tabular}

$\begin{array}{lll}50 & 60 & 70\end{array}$

Name

QASAWGRTVFQTSVQSLILGATIYLIGFDGTAVITRAVAADNGL

1. IBOV Ismalia2014 VP2 gene

2. $180 V$ Giza2008 VP2 gene

3. IBOV Giza2000 VP2 gene

4. IBOV S1.2012 VP2 gene

5. IBOV S2-2012 VP2 gene

6. IBOV S3-2012 VP2 gene

7. IBOV S4-2012 VP2 gene

8. IBOV S7-2013VP2 gene

9. IBOV $510-2013$ VP2 gene

10. IBDV S11-2013 VP2 gene

11. IBDV SV-G2 polyprotein gene

12. Bursavac

13. Bursine

14. DR-1

15. C. 278

16. UK661

17. $\mathrm{HK} 46$

18. $K 406 / 89$

19. K357/88

20. 078

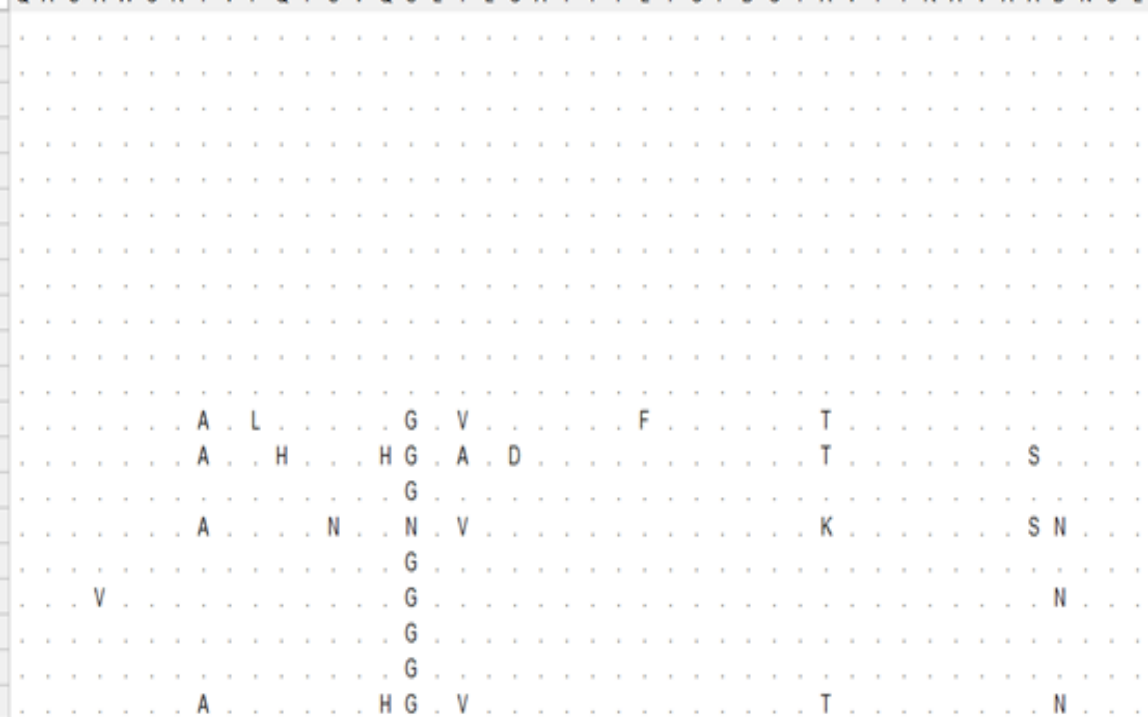

Figure (3): Deduced amino acid of different Egyptian IBDV compared with other strains. The horizontal line represents the sequence limits of VP2 gene. Dots indicate identical sequences.

\section{Phylogenetic tree}

As shown in figure (4) Ismailia isolate showed close relationships between the previously isolated
Egyptian IBDVs and they are clustered together while other classic attenuated vaccine strains (Bursavac and Bursine) are grouped in another clade. 


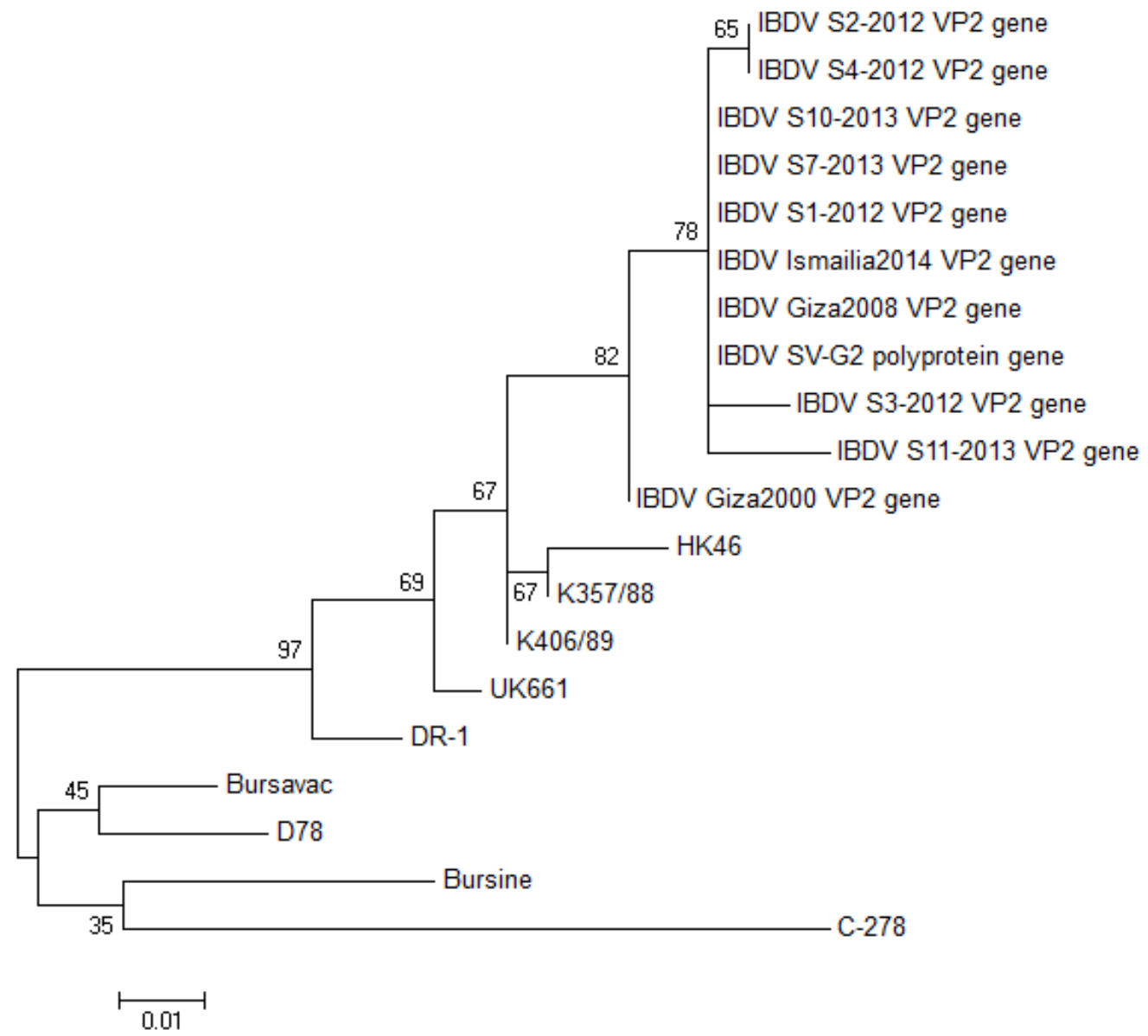

Figure (4): Phylogenetic tree based on a partial sequence of VP2 gene, showing the relationship between different IBDV isolates. The robustness of individual nodes of the tree was assessed using 1000 replications of bootstrap re-sampling of the originally aligned nucleotide sequences.

Table (1): Nucleotide similarity (\%) of the HVRs of VP2 of the examined strain with reference IBDV strains in Gen Bank.

\begin{tabular}{|c|c|c|c|c|c|c|c|c|c|c|c|c|c|c|c|c|c|c|c|c|}
\hline \multirow[b]{2}{*}{ Мห: } & \multirow[t]{2}{*}{ Arime } & \multirow{2}{*}{$\frac{D N}{d . N}$} & \multirow{2}{*}{ Anth } & \multicolumn{6}{|c|}{ 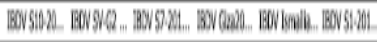 } & \multicolumn{2}{|c|}{ 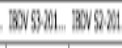 } & \multicolumn{3}{|c|}{ 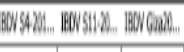 } & \multirow{2}{*}{$\frac{1166}{426}$} & \multirow{2}{*}{ (6) } & \multirow{2}{*}{$\frac{106601}{40104}$} & \multirow{2}{*}{ 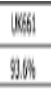 } & \multirow{2}{*}{$\frac{D N 1}{904}$} & \multirow{2}{*}{ 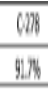 } \\
\hline & & & & 2064 & 20464 & $202 \pi$ & $20 \pi$ & $25 \pi$ & 2.56 & 2.24 & $215 \pi$ & 2.25 & Wh & Selt & & & & & & \\
\hline ON & 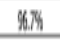 & & 2050 & 2146 & 1214 & 2215 & $23 x$ & 2015 & 2213 & 1214 & IIIS & 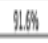 & $n_{2}, H_{H}$ & 214 & 1.665 & 2214 & $125 \pi$ & $21 \%$ & 20.0\% & 1.65 \\
\hline Awn & 1655 & 25 & & 1:2\% & 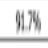 & $\| x$ & MIX & 12:35 & 133 & 1134 & NK & Mas & 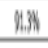 & M.X & Math & 1253 & $12 \pi$ & 1255 & 298 & 1336 \\
\hline 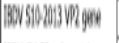 & 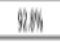 & Qis & $4 M$ & & 100 & 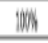 & IVA & 4as & Mon & 96 & M.3 & Min & WOS & 321 & 9115 & $9 . \mathrm{M}$ & Min & 93 & Sas & 9286 \\
\hline 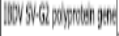 & 264 & 2) & 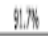 & 1004 & & $100 \pi$ & 1006 & 964 & $3: 60$ & \$2964 & 9.3 & $M M$ & $9 \pi$ & \$2: 24 & \$16 & $9 / \mathrm{M}$ & $91 / 4$ & Wh & Q864 & 92.26 \\
\hline 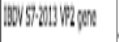 & $228 \mathrm{~K}$ & 2.14 & 14.3 & 104 & $100 \%$ & & $100 x$ & MAS & M.6 & 2196 & 928 & MSA & 9.96 & n2: & 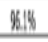 & 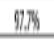 & 8113 & $9 ., A$ & 8.964 & 2023 \\
\hline 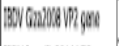 & 128K & Q1S & $\ln x$ & 106 & $10 \mathrm{~K}$ & (10N & & MAS & Mas & WW & NAS & MSA & MAK & MAN & W:15 & MN & MIS & $\ln x$ & 6964 & NAS \\
\hline 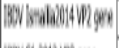 & 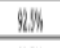 & thill & 4.W & Hill & WW & Men & Man & & 32.20 & 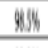 & 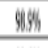 & MBO & 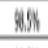 & 896 & Q16 & yith & 91,1 & xan & 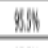 & HWW \\
\hline 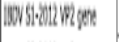 & 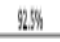 & 2415 & 9.36 & Mat & nas & 96 & Man & Mat & & 926 & 94 & 92 & $25 \%$ & 296 & \$1. & 9.1 .16 & $91 ., \pi$ & 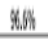 & 8SA & 践 \\
\hline 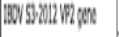 & $125 \pi$ & $2.4 \%$ & $0.3 \mathrm{~K}$ & 1060 & NWM & M.M & $x_{0}$ & 20.5 & n.2. & & 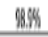 & 1950 & QHA & Q14. & 1023\% & MES & 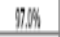 & 20.0 & 354 & Mas \\
\hline 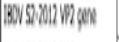 & Qis & Mis & $\operatorname{las}$ & MSN & N28 & NW & NW & Mas & Mas & MW & & MSK & Mist & 125N & ADN & naxt & nan & $k \pi$ & Bis & BAS \\
\hline 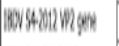 & 425 & then & 4. 45 & Hill & Math & 96 & Min & 3.86 & 324 & 9.54 & 由.M & & 睯 & 496 & 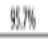 & 9 9.th & I.th & $9 h$ & 25:S & Hall \\
\hline 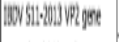 & H.X. & $W_{N}$ & 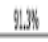 & xy & MX & 96 & 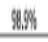 & 95 & 255 & 19.15 & 9.15 & 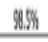 & & 2.5 & YHo & Y8S & $97 / 2$ & 8.85 & $9.7 \%$ & Bas \\
\hline 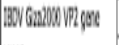 & 12.5 & 9.14 & 10.3 & M.2. & M26 & $M X$ & M.X. & M.S. & 19.4 & 1.14 & 1898 & MM & M.AS & & 1040 & 1959 & MOM & n., & HaW & N2\% \\
\hline K(if & H.W & 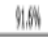 & Mak & Will & Wh & Wish & (kill & 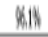 & kill & 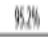 & $W M$ & SW & AWN & Man & & $M N$ & M.M & 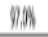 & 496 & $M N$ \\
\hline (09) & 90 & 2ill & 225 & $92 \pi$ & $9 \pi / \pi$ & 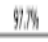 & $9, \pi$ & $9,4,4$ & Math & 2.06 & $910 \%$ & 91,6 & 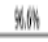 & sist & $9 \times 3$ & & 39 & 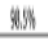 & 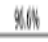 & BAS \\
\hline KWE & M.S & $2.25 \%$ & $\mathbb{R A N}$ & 8.16 & 2.15 & 9.5 & 8.15 & $9,1,3$ & M7. & 996 & 97.16 & 97.5 & $9 ., 4$ & 894 & 2836 & Mas & & 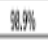 & 99.4 & B.8X \\
\hline UXal & 1336 & 20.14 & 12.5. & 19.4 & 19.4 & 17.24 & M.S4 & Man & Kath & Kas & 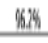 & 1660 & H.S.4. & 19.4 & 1984 & 1259 & NM & & 97.4 & 20.8 \\
\hline DN! & MAS & ASW & MAN & Mas & 9698 & W6 & Sin & 0.5 & 25.5 & 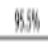 & 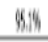 & Sis & $A N$ & MAN & Max & 1564 & 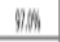 & 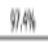 & & MPS \\
\hline (Wili & 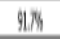 & $\| \mathbb{N}_{\pi}$ & D. WN & 9.26 & 9236 & 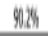 & 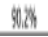 & BSW & Wat & 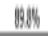 & 196 & 1964 & BSA & 9.26 & $W /$ & Bafla & Ban & 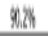 & MNA & \\
\hline
\end{tabular}




\section{DISCUSSION}

Because of the huge economic losses caused by this virus to poultry industry in Egypt, vaccination has therefore been applied intensively to control the infection. Kusk et al. (2005) found that effective vaccination depends on rapid and accurate diagnosis of the subtype present in a flock. Post-mortem findings reported in this study were characteristic to IBDV infection. Affected chickens showed congested bursa and sever peticheal hemorrhages on breast and thigh muscles, liver were congested, enlarged and mottled. A history of high mortalities (11-15\%) were recorded the rest of the examined flocks showed dehydration with slight muscular congestion and slightly enlarged bursa. The severity of these lesions depend upon virulence of the virus, age of birds and the presence or absence of passive immunity (Hassan, 2004; Rauw et al., 2007). It was suggested that pathogenesis and viral multiplication is more pronounced in very virulent strains comparing to the milder strains (Van den Berg et al., 2000).

Conventional RT-PCR has been useful and sensitive test in detecting IBDV serotypes, in the present study, RT-PCR was used to amplify a 620 bp fragment length within IBDV on VP2 gene from bursal samples (Abdel-Alim and Saif, 2001; Muller et al., 2003 ENREF 13). In this study 3 out of 20 (15\%) farm samples were positive by RT-PCR. Thus the other farms with the similar clinical signs suggesting that these signs may be attributed to other infectious or nutritional diseases other than IBDV infection.

The hypervariable region of VP2 amino acids contains the most informative genetic data regarding strain variability, it was chosen for sequence analysis to characterize IBDV strains molecularly, allowing analysis of variations that happen naturally or by attenuation in different strains, leading to changes in antigenicity and/or virulence (Banda et al., 2003; Jackwood and Wagner, 2007). In this study, a comparative alignment and phylogenetic analysis of the hypervariable domain of the VP2 showed that IBDV_Ismailia-2014 isolate shared significant similarity at the nucleotide level ranged from $99 \%$ to 100\% with other Egyptian vvIBDV (Giza 2008 and Giza 2000) (Abdel-Mawgod et al., 2014).

In this study, a method for IBDV typing was tested by the amplification of a 620-bp PCR product, which includes the hypervariable region of the VP2 gene (Ikuta et al., 2001). Further characterization of the isolates was based on the nucleotide sequence analysis of this PCR product. The sequence analysis have been included to determine the possible substitutions. Thus, nucleotide and amino acid sequence analysis are needed to completely identify this type of field isolate.
The results showed that there is only one base difference (C32G) between IBDV_Ismailia-2014 isolate and Giza 2008, IBDV_S10-2013, IBDV_S102013 and IBDV_S10-2013 isolates with 99.62\% identity. Also when compared with other circulating Egyptian strains as S1-2012, S2-2012, S3-2012, S42012 and S11-2013 our isolate showed very high similarity ranged from $99 \%$ to $100 \%$ which was characterized as very virulent (VVIBDV) (Sara Abdel-Mawgod et al., 2014) but when compared with vaccinal strains as Bursavac and Bursine it showed $92.45 \%$ and $91.32 \%$ identity respectively. It is indicated that Ismailia isolate showed close relationships between the previously isolated Egyptian IBDVs and they are clustered together while other classic attenuated vaccine strains (Bursavac and Bursine) were grouped in another clade. This indicates that the viruses isolated and circulating in Egypt showed higher similarities to each other while they showed lower similarities to the used vaccinal strains. Therefore, revising the vaccination programs in Egypt and using anew vaccinal strain is recommended.

\section{REFERENCES}

Abdel-Alim, G.A. and Saif, Y.M. (2001): Immunogenicity and antigenicity of very virulent strains of infectious bursal disease viruses. Avian diseases 45, 92-101.

Abdelmawgod, S.; Abdelsattar, A. and Hussien, H. (2014): Molecular genotypingof the infectious bursal disease virus (IBVD) isolated from broiler flocks in Egypt. International Journal of Veterinary Sceince and Medicine. 46 (52): 346- 522.

Banda, A.; Villegas, P. and El-Attrache, J. (2003): Molecular characterization of infectious bursal disease virus from commercial poultry in the United States and Latin America. Avian diseases 47, 87-95.

Bayliss, C.D.; Spies, U.; Shaw, K.; Peters, R.W.; Papageorgiou, A.; Muller, H. and Boursnell, M.E. (1990): A comparison of the sequences of segment A of four infectious bursal disease virus strains and identification of a variable region in VP2. The Journal of general virology 71 ( Pt 6), 1303-1312.

Cosgrove, A.S. (1962): An apparently new disease of chickens - avian nephrosis. Avian diseases 6, 385-389.

Coulibaly, F.; Chevalier, C.; Delmas, B. and Rey, F.A. (2010): Crystal structure of an Aquabirnavirus particle: insights into antigenic diversity and virulence determinism. J. Virol 84, 1792-1799.

El-Sergany, HA.; Ann Moursi; Saber, MS. and Mohamed, MA. (1974): A preliminary investigation on the occurrence of gunmboro 
disease in Egypt. Egypt. J. Vet. Sci. 11, 185-208.

Hassan, M.K. (2004): Very virulent infectious bursal disease virus in Egypt: epidemiology, isolation and immunogenicity of classic vaccine. Vet. Res. Commun 28, 347-356.

Ikuta, N.; El-Attrache, J.; Villegas, P.; Garcia, E.M.; Lunge, V.R.; Fonseca, A.S.; Oliveira, C. and Marques, E.K. (2001): Molecular characterization of Brazilian infectious bursal disease viruses. Avian diseases 45, 297-306.

Islam, M.R.; Rahman, S.; Noor, M.; Chowdhury, E.H. and Muller, H. (2012): Differentiation of infectious bursal disease virus (IBDV) genome segment B of very virulent and classical lineage by RT-PCR amplification and restriction enzyme analysis. Archives of virology 157, 333-336.

Jackwood, D.J. and Wagner, S. (2007): Genetic characteristics of infectious bursal disease viruses from four continents. Virology 365, 369-375.

Kusk, M.; Kabell, S.; Jorgensen, P.H. and Handberg, K.J. (2005): Differentiation of five strains of infectious bursal disease virus: development of a strain-specific multiplex PCR. Vet. Microbiol. 109, 159-167.

Li, Y.; Wu, T.; Cheng, X. and Zhang, C. (2009): Molecular characteristic of VP2 gene of infectious bursal disease viruses isolated from a farm in two decades. Virus Genes 38, 408-413.

Muller, H.; Islam, M.R. and Raue, R. (2003): Research on infectious bursal disease--the past, the present and the future. Vet. Microbiol 97, $153-165$
Rauw, F.; Lambrecht, B. and Van den Berg, T. (2007): Pivotal role of ChIFNgamma in the pathogenesis and immunosuppression of infectious bursal disease. Avian pathology: journal of the W.V.P.A 36, 367-374.

Singh, A.; Bedekar, M.K.; Sharma, R.; Sarkhel, B.C.; Singh, S. and Jain, S.K. (2012): Detection of very virulent infectious bursal disease virus from a field outbreak in Central India. Acta Vet Hung 60, 165-174.

Van den Berg, T.P.; Eterradossi, N.; Toquin, D. and Meulemans, G. (2000): Infectious bursal disease (Gumboro disease). Rev. Sci. Tech. 19, 509-543.

Wang, Y.; Kang, Z.; Gao, H.; Gao, Y.; Qin, L.; Lin, $H$.; Yu, F.; Qi, X. and Wang, X. (2011): A onestep reverse transcription loop-mediated isothermal amplification for detection and discrimination of infectious bursal disease virus. Virology journal 8, 108.

Wu, C.C.; Rubinelli, P. and Lin, T.L.9 (2007a): Molecular detection and differentiation of infectious bursal disease virus. Avian diseases 51, 515-526.

Wu, J.; Yu, L.; Li, L.; Hu, J.; Zhou, J. and Zhou, X. (2007b): Oral immunization with transgenic rice seeds expressing VP2 protein of infectious bursal disease virus induces protective immune responses in chickens. Plant Biotechnol J 5, 570-578.

Xu, X.G.; Tong, D.W.; Wang, Z.S.; Zhang, Q.; Li, Z.C.; Zhang, K.; Li, W. and Liu, H.J. (2011): Baculovirus virions displaying infectious bursal disease virus VP2 protein protect chickens against infectious bursal disease virus infection. Avian diseases 55, 223-229.

\section{الانتثار والوصف الجزيئى لفيروس الجمبورو فى مزارع الدواجن بالاسماعيلية نبفين رمزى ، شهيرة عبد الفتاح ، محد عبل الدايم}

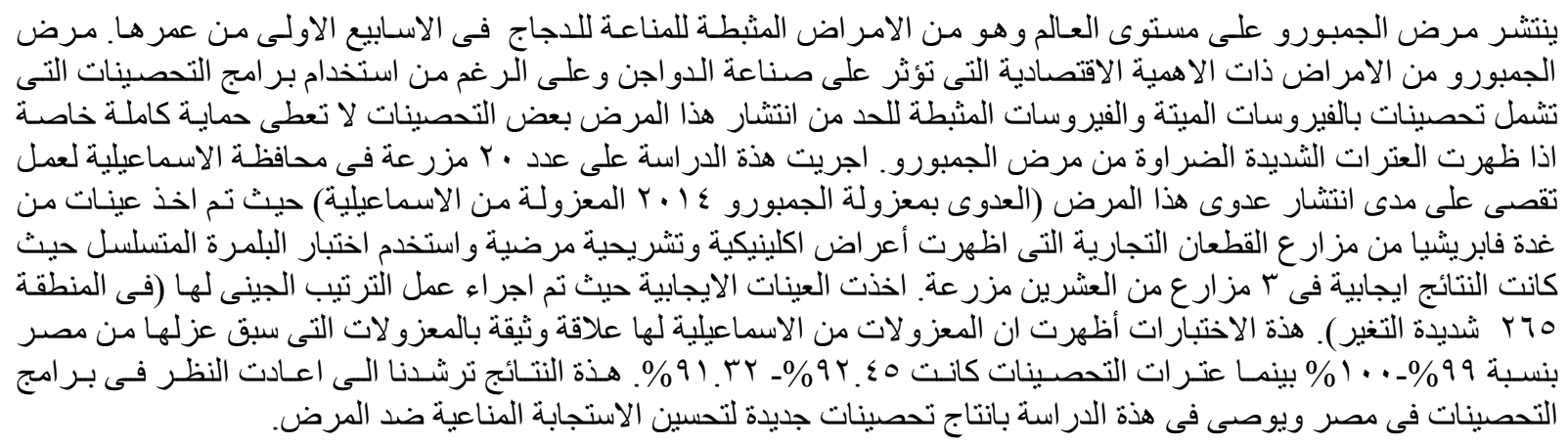

